\title{
Surgical revascularization for patients with diabetes: Do all roads lead to Rome?
}

\author{
Tomas A. Salerno, MD
}

The BARI 2D trial (Bypass Angioplasty Revascularization Investigation 2 Diabetes Trial) ${ }^{1}$ randomly allocated patients with type 2 diabetes and stable coronary artery disease (CAD) and evidence of myocardial ischemia to undergo, at the discretion of the treating physician, either early revascularization with a percutaneous intervention (PCI) or coronary artery bypass grafting (CABG) with optimal medical therapy (OMT), or OMT alone. Patients in the OMT group were also randomly assigned to insulinsensitizing strategy (metformin and thiazolidinedione) or an insulin-providing strategy (insulin or secretagogue.) The 5-year follow-up results for the entire cohort showed no significant differences in overall survival and major adverse cardiac and cerebrovascular event (death, myocardial infarction, and stroke) rate between early revascularization plus OMT versus OMT alone. The subgroup of patients with more extensive CAD, who were treated with early $\mathrm{CABG}$, however, had a lower rate of major adverse cardiac and cerebrovascular events than did those treated with OMT $(22.4 \%$ vs $30.5 \% ; P=.01)$; furthermore, the subgroup of patients with less extensive CAD, who were treated with early PCI, had similar outcomes to those treated with OMT alone. Likewise, there was no difference between the strategies of insulin sensitization and insulin provision. There was a significant crossover at 5 years, with $42.1 \%$ of the group initially assigned to OMT requiring clinically indicated revascularization with either PCI or CABG.

In this issue of The Journal of Thoracic and Cardiovascular Surgery, Sako and colleagues ${ }^{2}$ report on baseline demographic and clinical characteristics of the patient population in the BARI 2D trial that was enrolled in the surgical strata and provide technical details of the surgical procedures and the perioperative morbidity and mortality. This analysis ought to take into consideration the nonrandomized nature of the revascularization strategy selection, which shifted patients with more extensive $\mathrm{CAD}$ into the surgical treatment arm. Despite that, mean

\footnotetext{
From the Division of Cardiothoracic Surgery, University of Miami Miller School of Medicine, and Jackson Memorial Hospital, Miami, Fla.

Disclosures: Author has nothing to disclose with regard to commercial support. Received for publication Aug 22, 2014; accepted for publication Aug 22, 2014. Address for reprints: Tomas A. Salerno, MD, Division of Cardiothoracic Surgery (R-114), University of Miami Miller School of Medicine, PO Box 016960, JMH-East Tower Room 3072, Miami, FL 33136 (E-mail: tsalerno@med. miami.edu).

J Thorac Cardiovasc Surg 2014;148:1273-4

$0022-5223 / \$ 36.00$

Copyright (c) 2014 by The American Association for Thoracic Surgery http://dx.doi.org/10.1016/j.jtcvs.2014.08.034
}

left ventricular ejection fraction was $57.6 \%$, with only $17 \%$ of patients having a left ventricular ejection fraction less than $50 \%$. Furthermore, left anterior descending coronary artery stenosis greater than $50 \%$ was present in only $19.3 \%$ of this population. Nevertheless, surgical revascularization was superior to OMT alone, which is interesting considering that most patients in clinical practice are not referred for surgical revascularization unless proximal left anterior descending coronary artery disease is present and ejection fraction is depressed. Further, the total number of diseased vessels was small. Although 3.7 vessels were diseased when the cutoff for significance was set at $50 \%$, only 1.7 vessels were diseased when a cutoff of $70 \%$ was used for significance. This has major implications, considering that graft patency and clinical outcomes can be influenced by the degree of stenosis. More recent clinical trials have incorporated flow measurements with fractional flow reserve in the catheterization laboratory for clinical management of patients, as reported in the FAME trial. ${ }^{3}$ In reality, most of the $50 \%$ lesions and many of the $70 \%$ lesions were not ischemia producing. It is interesting that those patients still received the benefit of surgical revascularization, indicating the aggressive nature of even moderate CAD in the diabetic population and the rapid progression of disease. This issue is further supported by the fact that $42.1 \%$ of patients in the OMT-alone group eventually required revascularization within 5 years. Further research into defining OMT is needed to minimize progression of CAD in patients with diabetes.

With regard to the conduct and technical aspects of the procedures, there were so many variables involved in Sako and colleagues' report that it is not possible to come to any conclusion as to the best method, or whether there is even a difference in outcomes regardless of how the surgical procedures are performed. Of note is the fact that patients were accrued between 2001 and 2005. Considering techniques that are rarely used in North America (intermittent clamping, crystalloid cardioplegia), recent advances in technology, myocardial protection, off-pump CABG and others, and the heterogeneity of countries, centers and surgeons involved in the BARI 2D trial, it is rewarding to confirm that CABG still provided superior results in patients with diabetes. It may well be that superiority of surgical revascularization relies more on the fact that complete revascularization is always the goal, providing distal myocardial protection from ischemic insult in the long term, and is less dependent on the 
techniques or on the intraoperative details of the procedure as long as mortality remains low and arterial grafts (internal thoracic arteries) are used. More recent clinical trials, such as the SYNTAX (SYNergy between PCI with TAXUS and Cardiac Surgery) ${ }^{4}$ and FREEDOM (Future REvascularization Evaluation in patients with Diabetes mellitus: optimal management of Multivessel disease $)^{5}$ trials, have provided additional information about and confirmation of the benefits of CABG in the treatment of patients with diabetes who have CAD and ischemia. It is inevitable to conclude that the old adage still holds true, and all roads lead to Rome. CABG remains the best option for the treatment of patients with diabetes who have multivessel CAD and ischemia.

\section{References}

1. BARI 2D Study GroupFrye RL, August P, Brooks M, Hardison RM, Kelsey SF, MacGregor JM, et al. A randomized trial of therapies for type 2 diabetes and coronary artery disease. N Engl J Med. 2009;360:2503-15.

2. Sako EY, Brooks MM, Hardison RM, Schaff H, Frye R. Coronary artery bypass in patients with type 2 diabetes: experience from the Bypass Angioplasty Revascularization Investigation 2 Diabetes trial. J Thorac Cardiovasc Surg. 2014; 148:1268-72.

3. De Bruyne B, Pijls NH, Kalesan B, Barbato E, Tonino PA, Piroth Z, et al; FAME 2 Trial Investigators. Fractional flow reserve-guided PCI versus medical therapy in stable coronary disease. $N$ Engl J Med. 2012;367:991-1001.

4. Serruys PW, Morice MC, Kappetein AP, Colombo A, Holmes DR, Mack JM, et al; SYNTAX Investigators. Percutaneous coronary intervention versus coronaryartery bypass grafting for severe coronary artery disease. $N$ Engl J Med. 2009; 360:961-72.

5. Farkouh ME, Domanski M, Sleeper LA, Siami FS, Dangas G, Mack M, et al. FREEDOM Trial Investigators. Strategies for multivessel revascularization in patients with diabetes. N Engl J Med. 2012;367:2375-84. 